\title{
INVESTIGAÇÃO DA INFLUÊNCIA DA DOPAGEM DE CONDUTORES PROTÔNICOS DE CERATO DE BÁRIO
}

\section{INVESTIGATION OF THE INFLUENCE OF DOPING ON THE PROTON CONDUCTORS OF BARIUM CERATE}

\author{
H. B. B. C. DO NASCIMENTO ${ }^{1}$, M. J. GODINHO ${ }^{1,2}$ e R. H. G. A. KIMINAMI ${ }^{1}$ \\ ${ }^{1}$ Universidade Federal de São Carlos, Departamento de Engenharia de Materiais, Brasil \\ ${ }^{2}$ Universidade Federal de Goiás, Instituto de Química/RC, Brasil \\ E-mail: haruan.braga@dema.ufscar.br
}

\author{
article info \\ Article history: \\ Received 2017-09-04 \\ Accepted 2017-11-20 \\ Available online 2017-12-20
}

\begin{abstract}
PALAVRAS-CHAVE: Condutor Protônico; Cerato de Bário; Dopagem; Perovskita.
\end{abstract} KEYWORDS: Protonic Conductor; Barium Cerate; Doping; Perovskite

RESUMO: Com a crescente demanda para sistemas de energia eficientes e ambientalmente amigáveis, a conversão direta de combustíveis gasosos em eletricidade por células combustíveis recebeu tremenda atenção nas últimas décadas. Sendo assim, o estudo da sintese e sinterização do cerato de bário, um dos materiais mais promissores entre as células combustíveis, é de suma importância para aperfeiçoá-lo ao se obter novos pós e microestruturas diferenciadas, tornando essas células economicamente viáveis. A síntese pelo método de precursores poliméricos foi utilizada para o estudo da influência da dopagem do $\mathrm{BaCeO}_{3}$ com $\mathrm{Y}^{3+}$ como substituição parcial ao íon $\mathrm{Ce}^{4+}$. Os pós sintetizados foram caracterizados por análise térmica diferencial, difratometria de raios $X$, microscopia eletrônica de varredura, e por BET. Os resultados mostraram que foi possivel obter $\mathrm{BaCeO}_{3}$ dopadas com $\mathrm{Y}^{3+}$, que o método de síntese favoreceu na formação da fase a $900{ }^{\circ} \mathrm{C} / 2 \mathrm{~h}$ e que o efeito da dopagem com o $\mathrm{Y}^{3+}$ no cerato de bário $\left(\mathrm{BaCeO}_{3}\right)$ favoreceu na redução do tamanho médio das partículas de $182 \mathrm{~nm}$ (sem dopante) para $94 \mathrm{~nm}$ (com o 20\% $\mathrm{Y}^{3}$ ).

\begin{abstract}
With the increasing demand of efficient energy and environmentally friendly systems, the direct conversion of gaseous fuels into electricity by fuel cells received tremendous attention in the last decades. Given this, the barium cerate, one of the most promising materials among of fuel cells, synthesis and sinterization study is of utmost importance to improve it obtaining new powders and distinct microstructures, making these cells economically feasible. The polymeric precursor's method was used to study of doping of BaCeOs using $Y^{3+}$. The resulting material was characterized by DTA/TG, XRD, SEM and BET. The results indicate that $\mathrm{BaCeO}_{3}$ doping with $Y^{3+}$ were obtained successfully, the synthesis method favored the formation of the phase at 900 ${ }^{\circ} \mathrm{C} / 2 \mathrm{~h}$ and that the doping effect with $Y^{3+}$ was observed in reducing the mean particle size from $182 \mathrm{~nm}$ (without dopant) to $94 \mathrm{~nm}$ (with 20\% $\mathrm{Y}^{3}$ ).
\end{abstract}

\section{INTRODUÇÃO}

Devido à alta demanda energética, sistemas mais eficientes e com menor impacto ambiental vem sendo estudados com mais intensidade nos últimos anos (MEDVEDEV et al., 
2016; BABU et al., 2015) relacionados a conversão direta de combustíveis gasosos em eletricidade. Dentre esses sistemas, as Células a Combustíveis de Óxidos Sólidos (CCOS), construídas com eletrólitos condutores protônicos, têm atraído grande interesse pela possibilidade de uso de uma variedade de combustíveis $\left(\mathrm{CO}, \mathrm{CH}_{4} \mathrm{e} \mathrm{H}_{2}\right)$, alta eficiência e alta qualidade do calor de exaustão (YANG et al, 2014).

Materiais do tipo perovskita baseados em óxidos das famílias do $\mathrm{BaCeO}_{3}(\mathrm{BCO})$ e do $\mathrm{BaZrO}_{3}$ (BZO) são os óxidos condutores protônicos mais comumente investigados; em particular, $\mathrm{o} \mathrm{BaCeO}_{3}$ dopado mostra a maior condução protônica quando comparado com os outros (SUBRAMANIVAN et al., 2011). No entanto, sua elevada temperatura de síntese $\left(1150{ }^{\circ} \mathrm{C}\right)$ e relativamente baixa estabilidade química devem ser otimizadas para aplicação em CCOS comerciais que operem em temperaturas intermediárias.

Vários métodos têm sido utilizados na produção de eletrólitos sólidos de CCOS do tipo protônica, tais como os de mistura de óxido, sol-gel, Pechini, combustão e precursores poliméricos. Alguns destes métodos com os parâmetros de obtenção de temperatura e tempo de síntese dos materiais estão demostrados na Tabela 1.

Tabela 1 - Eletrólitos sólidos protônicos obtidos segundo a literatura.

\begin{tabular}{|c|c|c|c|c|}
\hline Material & Método & $\begin{array}{l}\text { Temperatura de } \\
\text { obtenção }\end{array}$ & Tempo & Referência \\
\hline $\begin{array}{l}\text { BCZO e } \\
\text { BCZYO }\end{array}$ & $\begin{array}{l}\text { Reação de estado } \\
\text { sólido }\end{array}$ & $1200-1300^{\circ} \mathrm{C}$ & $3 \mathrm{~h}$ & HAKIM 2015 \\
\hline BCZY & $\begin{array}{l}\text { Combustão } \\
\text { acetato- } \mathrm{H}_{2} \mathrm{O}_{2}\end{array}$ & $1100^{\circ} \mathrm{C}$ & $6 \mathrm{~h}$ & NASANI 2015 \\
\hline BCYO & Sol-gel & $1200^{\circ} \mathrm{C}$ & $24 h$ & $\begin{array}{c}\text { SUBRAMANIYAN } \\
2011\end{array}$ \\
\hline ВCYO e ВСРO & Sol-gel & $1000^{\circ} \mathrm{C}$ & - & LIU 2014 \\
\hline BCGO & $\begin{array}{l}\text { Processo dos } \\
\text { citratos }\end{array}$ & $1100^{\circ} \mathrm{C}$ & $4 \mathrm{~h}$ & $\begin{array}{c}\text { KHANDELWAL } \\
2011\end{array}$ \\
\hline BCSO & $\begin{array}{l}\text { Combustão ácido } \\
\text { cítrico-nitrato }\end{array}$ & $1100^{\circ} \mathrm{C}$ & $2 \mathrm{~h}$ & IWAHARA 1995 \\
\hline BSCYO & $\begin{array}{l}\text { Reação de estado } \\
\text { sólido }\end{array}$ & $1300^{\circ} \mathrm{C}$ & $10 \mathrm{~h}$ & LEE 2016 \\
\hline
\end{tabular}

O método mais utilizado na síntese de diferentes pós é pela mistura de óxidos realizados em moinhos de bolas, sendo depois levados a fornos em altas temperaturas para a calcinação, para que haja difusão dos íons (KREUER et al., 2011). Esse método, porém, envolve a calcinação em temperaturas muito elevadas e durante longos períodos (tipicamente acima de 10 horas), aumentando consideravelmente seu custo, dificultando seu uso comercialmente. Além disso, esse método também apresenta falta de reprodutibilidade, partículas grandes e pouca homogeneidade química. Devido a essas desvantagens, os métodos químicos se mostram de extrema importância para a produção economicamente viável de células combustíveis à base de cerato de bário.

Outro método comumente utilizado na preparação do cerato de bário é a decomposição térmica de sais precursores, como carbonatos. Ultimamente outras técnicas de síntese têm 
sido utilizadas na preparação de partículas de diâmetro nanométrico, sendo a dos precursores poliméricos a que demostra ser a mais promissora para síntese em escala superior à laboratorial. Isso se deve à homogeneidade da distribuição dos cátions e da estequiometria desejada e à razão custo/benefício, boa reprodutibilidade, alto rendimento e por permitir a obtenção de quantidades em grande escala (GONÇALVES et al., 2015). Esse método também possibilita a síntese de perovskitas com diferentes dopantes. A temperatura empregada nesse processo é menor que a usada na mistura de óxidos, diminuindo o gasto energético e consequentemente os custos. Além disso, a distribuição de cada componente é homogênea, pois os íons metálicos são completamente dissolvidos na resina polimérica durante o processo, resultando em pós de alta homogeneidade composicional e uma distribuição de tamanho de partículas na escala de nanômetros, ajudando no processo de sinterização, podendo-se produzir cerâmicas com alta densidade e alta pureza (KAKIHANA et al., 1999; PECHINI, 1967; GODINHO et al., 2003; ZAMPIERI et al., 2006).

O método dos precursores poliméricos, difere um pouco da rota proposta por Pechini (FENG et al., 2015), onde está última, utiliza-se uma rede polimérica pronta para a solubilização dos cátions. Na rota por precursores poliméricos, ocorre antes a síntese de compostos de coordenação solúveis e, posteriormente, esses compostos de coordenação são polimerizados com a adição de um agente polimerizante, como um poliálcool, normalmente, o etilenoglicol. (MEDVEDEV et al., 2016). Nesse método a quelação é realizada quando os cátions metálicos e o ácido cítrico são dissolvidos em uma solução aquosa. Desta forma, após a dissolução do ácido cítrico em água, a solução tem seu $\mathrm{pH}$ corrigido para um valor neutro $(\mathrm{pH}=7)$ para o desprotonamento das carboxilas, fazendo com que os cátions metálicos ocupem a posição dos íons $\mathrm{H}^{+}$na rede, formando um complexo ácido cítrico-metal (KREUER et al., 2011). Na presença de etilenoglicol ocorre uma reação de poliesterificação do complexo, resultando em éster e água. Esse polímero mantém a distribuição homogênea e aleatória dos íons metálicos, sendo essa uma das vantagens do método dos precursores poliméricos.

Nas Células a Combustíveis de Óxidos Sólidos, o combustível, geralmente gás hidrogênio, se oxida no anodo, separando-se em prótons e elétrons, segundo a Equação 1:

$$
H_{2} \rightarrow 2 H^{+}+2 e^{-}
$$

Os elétrons então fluem por um circuito externo e entra em contato com o gás oxigênio no cátodo, como representando na equação 2 , abaixo:

$$
\frac{1}{2} \mathrm{O}_{2}+2 e^{-} \rightarrow \mathrm{O}^{2-}
$$

A reação global é a soma das equações (1) e (2), resultando na equação (3):

$$
\mathrm{H}_{2}+\frac{1}{2} \mathrm{O}_{2} \rightarrow \mathrm{H}_{2} \mathrm{O}
$$

O fato de o único resíduo desse processo ser água demonstra que a geração de energia elétrica por CCOS é ambientalmente favorável, já que não emite poluentes. Para que esse processo ocorra, deve haver a migração dos íons através do eletrólito, indo de um eletrodo ao 
outro. Caso o íon a migrar seja o $\mathrm{O}^{2-}$, a condução é iônica; caso seja o $\mathrm{H}^{+}$a migrar, será condução protônica, essa seletividade é relativa ao tipo de óxido utilizado como eletrólito.

A condução protônica é mais vantajosa que a iônica em CCOS, pois a água se forma no cátodo, evitando a diluição do combustível, aumentando a eficiência da célula e outro fator é a diminuição da temperatura de operação das células.

A condução protônica de óxidos do tipo perovskita é devido à presença de defeitos catiônicos em sua rede, criados pela dissociação da água absorvida na presença de vacâncias de oxigênio (NORBY 1999, LIU et al 2014). Na notação de Kröger-Vink, tal reação é escrita como na Equação 4:

$$
\mathrm{H}_{2} \mathrm{O}+\mathrm{V}^{\bullet \bullet}{ }_{\mathrm{O}}+\mathrm{O}^{x} \mathrm{O} \rightarrow 2 \mathrm{OH}^{\bullet} \mathrm{O}
$$

onde, $\mathrm{O}^{\mathrm{x}}{ }_{\mathrm{O}}$ representa o íon oxigênio na sua posição normal na rede cristalina, $\mathrm{V}^{*}{ }_{\mathrm{o}}$ é a vacância de oxigênio e $\mathrm{OH}_{0}^{\circ}$ é o portador de carga protônico formado no interior da estrutura pela associação à subrede do oxigênio. $O$ salto de prótons entre vacâncias de oxigênio adjacentes é a origem da condutividade protônica. (ZAMPIERI et al).

Essa dopagem produz vacâncias de oxigênio de acordo com a equação 5, utilizando a notação de Kröeger e Vink, em que $\mathrm{R}$ representa o cátion trivalente, por exemplo o $\mathrm{Y}^{3+}, \mathrm{O}^{{ }_{0}}$ representa o oxigênio em sua posição na rede e $\mathrm{V}^{*}$ o representa a vacância de oxigênio gerada.

$$
\mathrm{R}_{2} \mathrm{O}_{3} \rightarrow 2 \mathrm{R}^{\prime}{ }_{\mathrm{Ce}}+3 \mathrm{O}^{\mathrm{x}} \mathrm{O}+\mathrm{V}^{\bullet \bullet} \mathrm{O}
$$

Quando materiais desse tipo entram em contato com o vapor de água da atmosfera, as vacâncias de oxigênio geradas são preenchidas com hidroxilas originárias das moléculas de água, segundo a equação 6:

$$
\mathrm{H}_{2} \mathrm{O}_{(g)}+\mathrm{V}^{\bullet \bullet}{ }_{\mathrm{O}}+\mathrm{O}^{x} \mathrm{O} \rightarrow 2 \mathrm{OH}^{\bullet} \mathrm{O}
$$

A condução dos prótons é realizada através destes grupos hidroxila inseridos na rede cristalina do material, fazendo com que o próton migre de uma hidroxila para a subsequente, gerando uma corrente protônica. Este mecanismo de condução protônica é conhecido como mecanismo de Grotthuss.

Desta forma, o objetivo deste trabalho foi a investigação da influência da dopagem em condutores protônicos de cerato de bário utilizando $\mathrm{Y}^{3+}$ como substituição parcial ao íon $\mathrm{Ce}^{4+}$.

\section{MATERIAIS E MÉTODOS}

Eletrólitos de $\mathrm{BCO}$ puros e dopados com Y (BCYO), foram preparados por meio do método dos precursores poliméricos, sintetizando-se $\mathrm{BaCe}_{1-\mathrm{x}} \mathrm{Y}_{\mathrm{x}} \mathrm{O}_{3}(\mathrm{com} 0 \leq \mathrm{x} \leq 20)$. Os sais precursores utilizados foram o $\mathrm{Ba}\left(\mathrm{NO}_{3}\right)_{2}, \mathrm{Ce}\left(\mathrm{NO}_{3}\right)_{3} \cdot 6 \mathrm{H}_{2} \mathrm{O}$ e $\mathrm{Y}\left(\mathrm{NO}_{3}\right)_{3} \cdot \mathrm{H}_{2} \mathrm{O}$ todos de grau analítico.

Primeiramente foram sintetizados os citratos dos cátions em separado através da 
dissolução, na proporção de 3:1 de ácido cítrico:cátion metálico. Após, foram misturados os citratos metálicos em proporções apropriadas, e o $\mathrm{pH}$ foi ajustado para $\mathrm{pH}=7$ através da adição de hidróxido de amônio. A resina polimérica foi então formada com a adição de etilenoglicol à solução resultante, sempre sob agitação e aquecimento de $70{ }^{\circ} \mathrm{C}$. A resina polimérica foi pré-calcinada, obtendo-se o "puff" (material carbonizado) e este foi desaglomerado, obtendo-se o material desejado rico em carbono. $\mathrm{O}$ pó resultante foi então calcinado em diferentes temperaturas para investigação da temperatura de formação da fase desejada.

Para investigar a formação de fases e morfologia dos pós foram realizadas as caracterizações por difração de raios X em um difratômetro Siemens D5005 com radiação K $\alpha$ do cobre $(\lambda=1,5406 \AA)$, microscopia eletrônica de varredura em um microscópio FEI Inspect S 50, ATD/TG (Netzsch STA-409) e a área superficial específica foi determinada utilizando o método de Brunauer, Emmett and Teller (BET) em um equipamento Micromeritics ASAP 2020. O tamanho médio de cristalito foi calculado utilizando a equação de Scherrer.

\section{RESULTADOS E DISCUSSÃO}

A identificação da fase cristalina do BCO puro e dopado com ítrio foi obtida através da caracterização por difração de raios $\mathrm{X}$, após calcinação a $800{ }^{\circ} \mathrm{C}, 900{ }^{\circ} \mathrm{C}$ e $1000{ }^{\circ} \mathrm{C}$ para as amostras puras e dopadas, com taxa de aquecimento de $5{ }^{\circ} \mathrm{C} / \mathrm{min}$, durante $2 \mathrm{~h}$ em forno do tipo mufla.

Os resultados de $\mathrm{DRX}$ do $\mathrm{BaCeO}_{3}(\mathrm{BCO})$ estão apresentados na Figura 1a, e do $\mathrm{BaCe}_{0,8} \mathrm{Y}_{0,2} \mathrm{O}_{3}(\mathrm{BCYO} 20)$ na Figura $1 \mathrm{~b}$, onde foi possível identificar a formação da fase romboédrica do tipo perovskita a partir de $800{ }^{\circ} \mathrm{C}$, de acordo com a ficha cristalográfica JCPDS 00-001-0803, tanto para o sistema puro quanto para o dopado com $20 \% \mathrm{Y}$.

Ao se utilizar o método dos precursores poliméricos, a temperatura de síntese foi de apenas $1000{ }^{\circ} \mathrm{C}$ para o $\mathrm{BaCeO}_{3}$ puro (Figura 1a) e $900{ }^{\circ} \mathrm{C}$ para o $\mathrm{BaCe}_{0,8} \mathrm{Y}_{0,2} \mathrm{O}_{3}$ (Figura 1b), inferior aos métodos apresentados na Tabela 1, demonstrando o efeito da dopagem $\mathrm{Y}^{3+}$ como substituição parcial ao íon $\mathrm{Ce}^{4+}$ na diminuição da temperatura de síntese. Outro fator importante a ser destacado foi o tempo de calcinação ser apenas de 2 horas, frente aos tempos maiores utilizados nos outros métodos de síntese (Tabela 1). A escolha do dopante de ítrio está de acordo com dados da literatura, onde a substituição é favorecida devido a proximidade de raios iônicos entre $\mathrm{Y}^{3+}=0,900 \mathrm{~A}$ e $\mathrm{Ce}^{4+}=0,870 \mathrm{~A}$ (KNIGTH, 1992). Outro fator importante é que embora as vacâncias possam ser criadas intrinsecamente em um processo favorecido pelo aumento de temperatura, a adição de um dopante trivalente também leva à criação de vacâncias extrinsecamente. Desta forma, a criação de um número de vacâncias adequado colabora no aumento da condutividade protônica do eletrólito sólido (GU et al, 2013). Contudo, a determinação do teor de dopante segue o compromisso de manter-se abaixo do limite de solubilidade para a formação de solução sólida e de criar maior número de vacâncias e portadores de carga. 

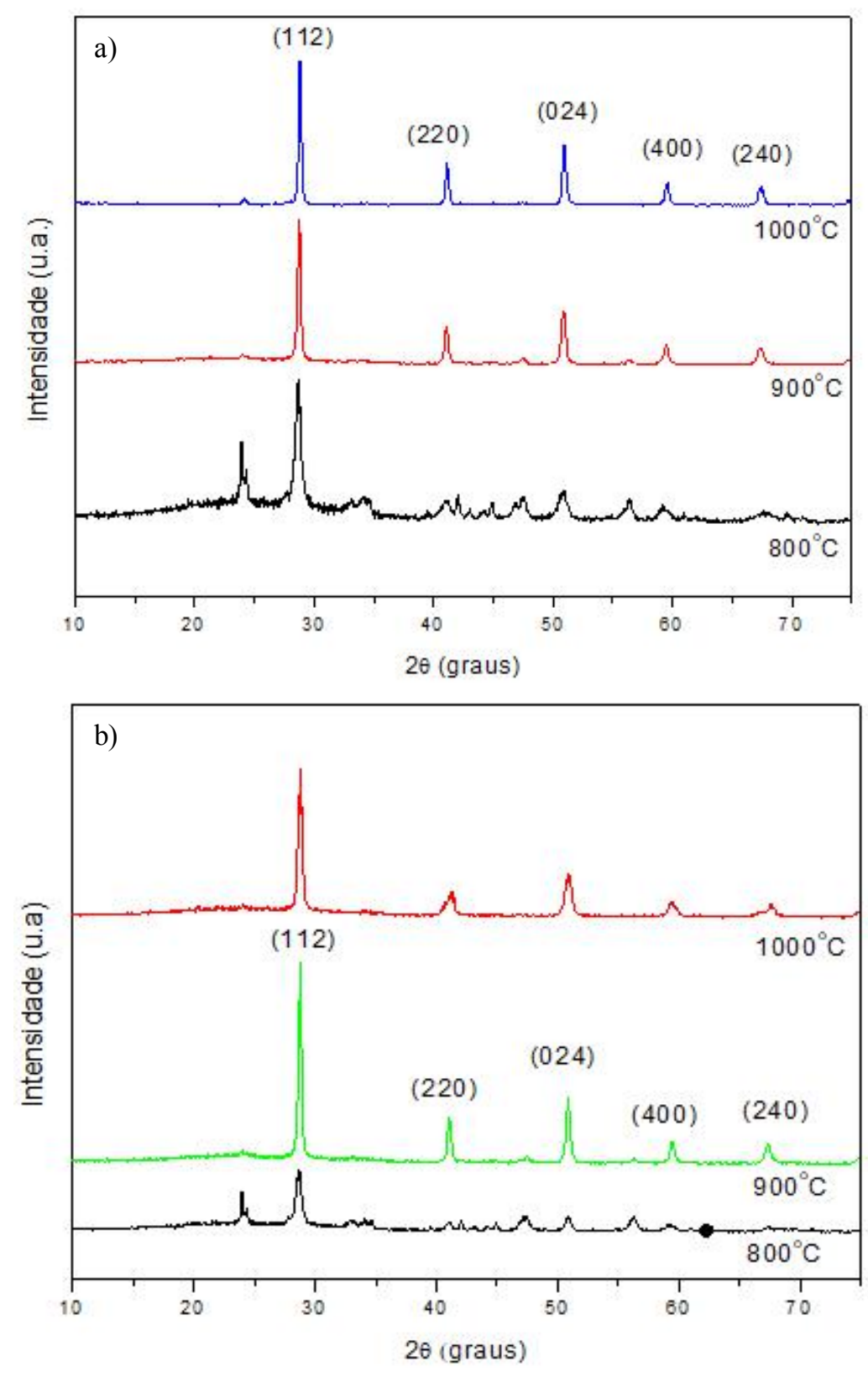

Figura 1- (a) Difratogramas do $\mathrm{BaCeO}_{3}$ e (b) $\mathrm{BaCe}_{0,8} \mathrm{Y}_{0,2} \mathrm{O}_{3}$.

O tamanho médio do cristalito foi calculado a partir da equação de Scherrer (equação 7) e os dados de difração de raios $\mathrm{X}$, que relacionam o tamanho aparente do cristalito $L$ com a largura integral $\beta_{\mathrm{tc}}$ na escala $2 \theta$ (rad). A partir dos dados calculados foi observado uma diminuição de $44,3 \mathrm{~nm}$ para $28,2 \mathrm{~nm}$ com relação ao BCO puro para o BCYO com $20 \% \mathrm{Y}$, respectivamente.

$$
L=\lambda /\left(\beta_{t c} \cos \theta\right)
$$



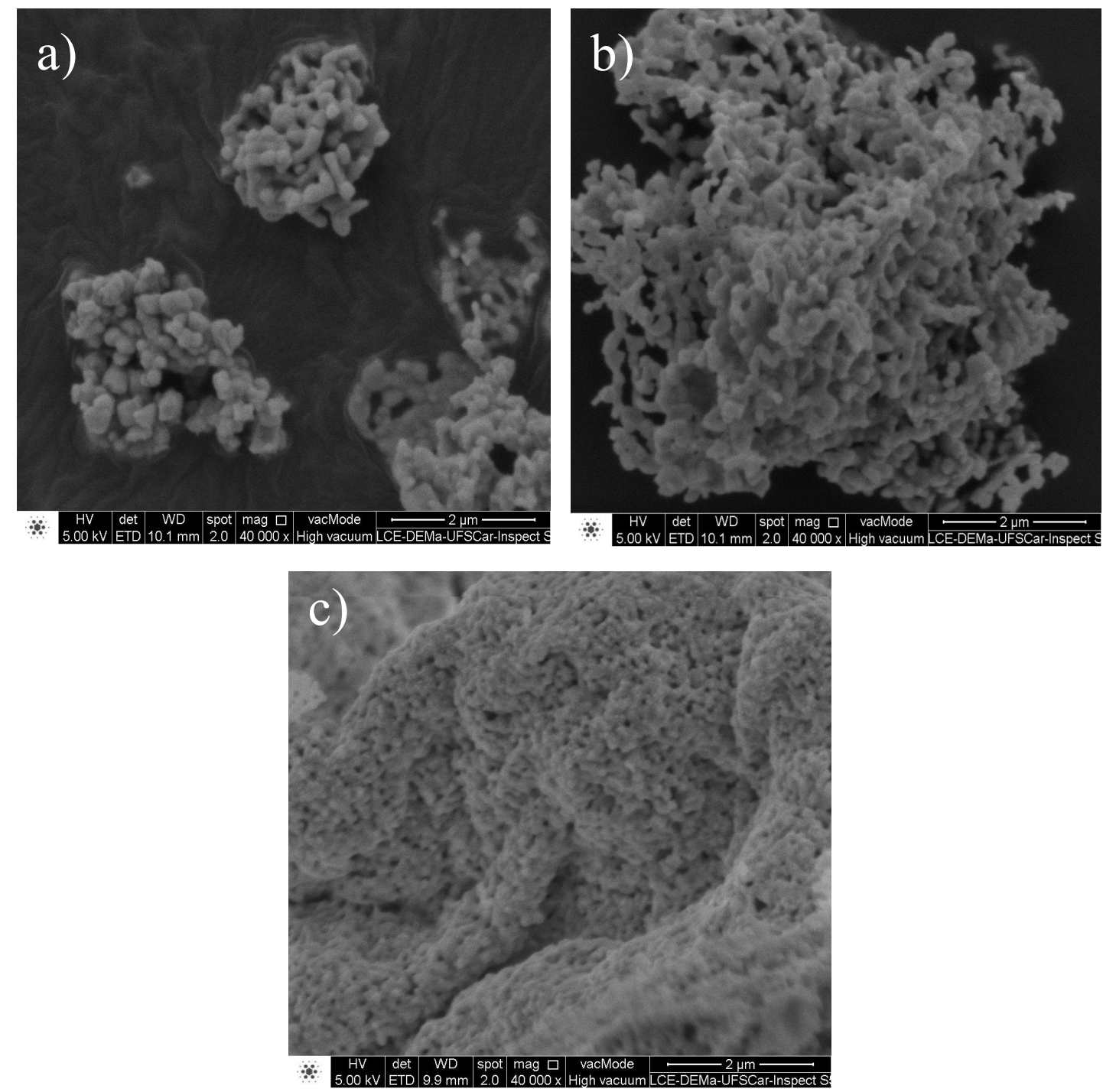

Figura 2- Micrografias das amostras de (a) $\mathrm{BaCeO}_{3},(\mathrm{~b}) \mathrm{BaCe}_{0,9} \mathrm{Y}_{0,2} \mathrm{O}_{3} \mathrm{e}$ (c) $\mathrm{BaCe}_{0,8} \mathrm{Y}_{0,2} \mathrm{O}_{3}$.

Na Figura 2 são mostradas as imagens de microscopia eletrônica de varredura (MEV) das amostras com e sem dopagem com ítrio, onde é possível observar uma diminuição do tamanho médio das partículas. Essa diminuição do tamanho das partículas com o aumento da quantidade de dopante pode ser relacionada ao aumento de distorções na rede cristalina, a qual dificulta o crescimento de partícula.

A Tabela 2 apresenta os resultados do tamanho médio das partículas calculado pelo método dos interceptos. Observa-se nesse caso redução de $182 \mathrm{~nm}$ do BCO puro para $94 \mathrm{~nm}$ para o BCYO20. Também houve maior aglomeração das partículas, devido à maior área superficial de contato, devido às forças atrativas entre as partículas. 
Tabela 2: Tamanho médio de partícula e área superficial

\begin{tabular}{ccc}
\hline Material & $\begin{array}{c}\text { Tamanho médio de } \\
\text { partícula }(\mathbf{n m})\end{array}$ & Área Superficial $\left(\mathbf{m}^{\mathbf{2}} / \mathbf{g}\right)$ \\
\hline BCO & 182 & 4,8 \\
\hline BCYO10 & 133 & 7,1 \\
\hline BCYO20 & 94 & 8,2 \\
\hline
\end{tabular}

Ao se comparar os resultados obtidos neste trabalho, da temperatura e do tempo necessário de calcinação das amostras de $\mathrm{BCO}$ a $1000{ }^{\circ} \mathrm{C}$ e $\mathrm{BCYO} 20$ a $900{ }^{\circ} \mathrm{C}$ com os da literatura pôde-se constatar que, a formação da fase em todas as amostras, ocorreu em temperaturas e tempos inferiores, quando comparados com os métodos reportados na literatura (Tabela 1). Os valores da área superficial foram $8,2 \mathrm{~m}^{2} / \mathrm{g}$ para o BCYO20, $7,1 \mathrm{~m}^{2} / \mathrm{g}$ para o $\mathrm{BCYO} 10$ e $4,8 \mathrm{~m}^{2} / \mathrm{g}$ para o $\mathrm{BCO}$, corroborando os resultados anteriormente discutidos.

\section{CONCLUSÃO}

A partir do difratograma de raios $\mathrm{X}$ constatou-se que a fase pura do $\mathrm{BaCeO}_{3}$ foi obtida pelo método dos precursores poliméricos em temperaturas e tempos inferiores aos reportados na literatura, e que o efeito da dopagem com o $\mathrm{Y}^{3+}$ como substituição parcial ao íon $\mathrm{Ce}^{4+}$ favoreceu na redução do tamanho médio das partículas de $182 \mathrm{~nm}$ (sem dopante) para $94 \mathrm{~nm}$ (com o $20 \% \mathrm{Y}^{3+}$ ). O método favoreceu na obtenção dos pós puros, cristalinos e homogêneos.

\section{AGRADECIMENTOS}

À FAPESP e ao CNPq pelo apoio financeiro e bolsas (Processos: 2016/20512-4, 485518/2013-09 e 431304/2016-5).

\section{REFERÊNCIAS}

BABU A.S., BAURI R.; Phase evolution and morphology of nanocrystalline $\mathrm{BaCe}_{0.9} \mathrm{Er}_{0.1} \mathrm{O}_{3-\delta}$ proton conducting oxide synthesised by a novel modified solution combustion route. Journal of Physics and Chemistry of Solids. v.87, p.80-86, 2015.

GODINHO M.J., BUENO P.R.; ORALANDI M.O.; LEITE E.R.; LONGO E. Ionic conductivity of $\mathrm{Bi}_{4} \mathrm{Ti}_{0.2} \mathrm{~V}_{1.8} \mathrm{O}_{10.7}$ polycrystalline ceramics obtained by the polymeric precursor route. Materials Letters. v. 57, p. 2540-2544, 2003.

GONÇALVES R.F., LIMA A.R.F., GODINHO M.J. MOURA A.P., ESPINOSA J. LONGO E., MARQUES A.P.A. Synthesis of $\mathrm{Pr}^{3+}$-doped $\mathrm{CaTiO}_{3}$ using polymeric precursor and microwave-assisted hydrothermal methods: A comparative study. Ceramics International, v. 41, p.12841-12848, 2015.

GU Y., LIU Z., OUYANG J., YAN F. and ZHOU Y. Structure and electrical conductivity of $\mathrm{BaCe}_{0.85} \mathrm{Ln}_{0.15} \mathrm{O}_{3-\mathrm{d}}$ ( Ln- Gd, Y, Yb) ceramics. Electrochimica Acta, v. 105, p. 547-553, 2013.

HAKIM M., YOO C.Y. JOO J.H., YU H. Enhanced durability of a proton conducting oxide fuel cell with a purified yttrium-doped barium zirconate-cerate electrolyte. Journal of Power Sources. v. 278, p.320-324, 2015. 
IWAHARA H. Technological challenges in the application of proton conducting ceramics. Solid State Ionics. v. 77, p.289-298, 1995.

KAKIHANA M., YOSHIMURA M. Synthesis and characteristics of complex multicomponent oxides prepared by polymer complex method. Bulletin of the Chemical Society Japan. v.72, p.1427-1443, 1999.

KHANDELWAL M., VENKATASUBRAMANIAN A. PRASANNA T.R.S., GOPALAN P. Correlation between microstructure and electrical conductivity in composite electrolytes containing Gd-doped ceria and Gd-doped barium cerate. Journal of the European Ceramic Society, v. 31, n. 4, p.559-568, 2011.

KNIGTH K. S., SOAR M. and BONANOS N. Crystal structures of gadolinium and yttriumdoped barium cerate, Journal of Materials Chemistry., v. 2, p. 709-718, 1992.

KREUER K.D., ADAMS S., MÜNCH W. FUCHS A., KLOCK U., MAIER J. Proton conducting alkaline earth zirconates and titanates for high drain electrochemical applications. Solid State Ionics. v. 145, p. 295-306, 2001.

LEE T. R., LIMA D. K., SINGH B., SONG S. J. Study of mass transport kinetics in codoped $\mathrm{Ba}_{0.9} \mathrm{Sr}_{0.1} \mathrm{Ce}_{0.85} \mathrm{Y}_{0.15} \mathrm{O}_{3-\delta}$ by electrical conductivity relaxation. Solid State Ionics v.289, p. 9-16, 2016.

LIU Y. RAN R., LI S., JIAO Y., TADE M.O., SHAO Z. Significant performance enhancement of yttrium-doped barium cerate proton conductor as electrolyte for solid oxide fuel cells through a Pd ingress-egress approach. Journal of Power Sources. v. 257, p.308-318, 2014.

MEDVEDEV D.A., LYAGAEVA J.G.; GORBOVA E.V.; DEMIN A.K.; TSIAKARAS P. Advanced materials for SOFC application: Strategies for the development of highly conductive and stable solid oxide proton electrolytes. Progress in Materials Science. v.75, p.38-79, 2016.

NASANI N, RAMASAMY D., MIKHALEV S, KOVALESVSKY A.V. FAGG D.P. Fabrication and electrochemical performance of a stable, anode supported thin $\mathrm{BaCe}_{0.4} \mathrm{Zr}_{0.4} \mathrm{Y}_{0.2} \mathrm{O}_{3-\delta}$ electrolyte Protonic Ceramic Fuel Cell. Journal of Power Sources, v. 278, p.582-589, 2015.

NORBY T., Solid-state protonic conductors: principles, properties, progress and prospects. Solid State Ionics. v. 125, p 1-11, 1999.

PECHINI M.P., U.S. Patent. No 3, 330 (1967) 697.

SUBRAMANIYAN A., TONG J., O'HAYRE R.P., SAMMES N.M. ubramaniyan, Archana et al. Sintering Studies on $20 \mathrm{~mol} \%$ Yttrium-Doped Barium Cerate. Journal of The American Ceramic Society. v. 94, n. 6, p.1800-1804, 2011.

YANG, N., TEBANO A., CASTRO D., BALESTRINO G., D’EPIFANIO A. LICOCCIA S., DI BARTOLOMEO E. Deposition and electrochemical characterization of Yttrium doped Barium cerate and zirconate heterostructures. Thin Solid Films. v.562, p.264268, 2014.

ZAMPIERI M., LAZARO S.R., PASKOCIMAS C.A., FERREIRA A.G., LONGO E. VARELA J.A. Structural Analysis of Ti and Pb Citrate Using NMR and FT-Raman Signals and Quantum Mechanics Simulations. Journal of Sol-Gel Science and Technology. v.37, p.9-17, 2006. 